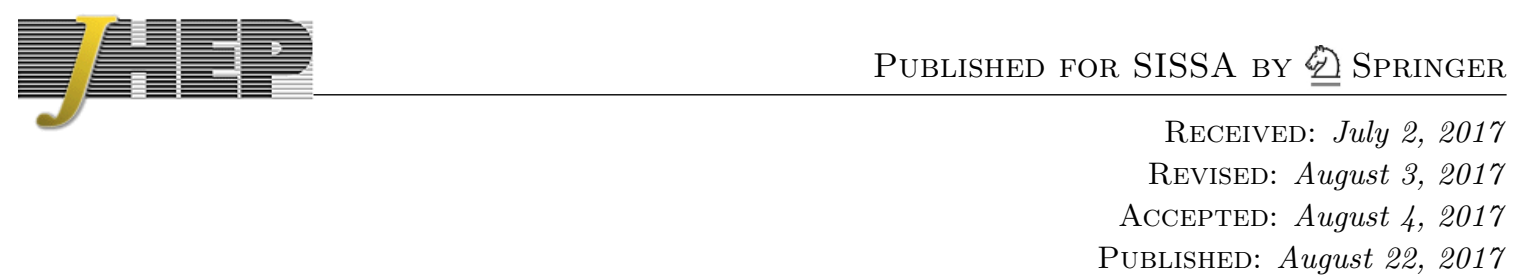

\title{
Enlarging regions of the MSSM parameter space for large $\tan \beta$ via SUSY decays of the heavy Higgs bosons
}

\author{
Anibal D. Medina ${ }^{a, b}$ and Michael A. Schmidt ${ }^{c}$ \\ ${ }^{a}$ Institut de Physique Théorique, Université Paris Saclay, \\ CNRS, CEA, F-91191 Gif-sur-Yvette, France \\ ${ }^{b}$ IFLP, CONICET-Departamento de Física, Universidad Nacional de La Plata, \\ C.C 67, 1900 La Plata, Argentina \\ ${ }^{c} A R C$ Centre of Excellence for Particle Physics at the Terascale, \\ School of Physics, The University of Sydney, \\ Physics Road, NSW 2006, Australia \\ E-mail: anibal.medina@fisica.unlp.edu.ar, \\ michael.schmidt@sydney.edu.au
}

AbStract: In the Minimal Supersymmetric Standard Model (MSSM) searches for the heaviest CP-even and CP-odd Higgs $H, A$ to tau-lepton pairs severely constrain the parameter region for large values of $\tan \beta$ and light Higgs bosons $H, A$. We demonstrate how the experimental constraint can be avoided by new decays to light third-generation sfermions, whose left-right couplings to $H$ can be maximised in regions of large trilinear couplings $A_{b}, A_{\tau}$ for sbottoms and staus, or large supersymmetric (SUSY) Higgs mass $\mu$ for stops. Due to the $\tan \beta$-enhancement in the production cross-sections via gluon-fusion and in association with bottom-quark pairs for $H$ and $A$, we find that down-type sfermions, in particular, sbottoms perform a better job in allowing more parameter space than up-type sfermions such as stops, which require much larger values of $\mu$ to compensate for $\tan \beta$. Vacuum stability as well as flavour observables constraints and direct searches for SUSY particles are imposed. We also associate the lightest CP-even Higgs with the observed $125 \mathrm{GeV}$ SM-like Higgs and impose the experimental constraints from the LHC.

KeYwords: Supersymmetry Phenomenology

ARXIV EPRINT: 1706.04994 


\section{Contents}

1 Introduction 1

2 Analytical motivation 2

3 Light sbottoms 4

3.1 Numerical scan 4

$\begin{array}{llr}3.2 & \text { Results } & 6\end{array}$

4 Light staus $\quad 9$

4.1 Numerical scan 9

$\begin{array}{lll}4.2 & \text { Results } & 10\end{array}$

5 Light stops 12

6 Conclusion 12

\section{Introduction}

More than one Higgs doublet is expected in many theories beyond the Standard Model (SM). The most economical and well-studied supersymmetric extension of the SM, the Minimal Supersymmetric Standard Model (MSSM), contains a type-II two Higgs doublet system due to holomorphicity in its electroweak symmetry breaking sector (EWSB). If CP is a good symmetry of the Higgs sector, the scalar Higgs spectrum consists of two CP even Higgs bosons $h$ and $H$, one CP odd Higgs $A$ and charged Higgs pair $H^{ \pm}$; the lightest CPeven Higgs $h$ is most easily identified with the $125 \mathrm{GeV}$ SM-like Higgs resonance discovered at the LHC. On the other hand, heavier neutral Higgs bosons are being searched for at the LHC via their decay into a pair of tau-leptons and strong constraints are put on the allowed masses as a function of the ratio of the Higgs doublet vacuum expectation values (vev) $\tan \beta \equiv\left\langle H_{u}\right\rangle /\left\langle H_{d}\right\rangle=v_{u} / v_{d}$. In particular, the latest CMS [1] and ATLAS [2] results show that if only decays to SM fermions and gauge bosons are considered, then $m_{H} \approx m_{A}>500 \mathrm{GeV}$ for $\tan \beta \gtrsim 20$, ruling out regions of large $\tan \beta$ and moderate $m_{A}$. These regions however are very appealing since for $\tan \beta \gg 1$ there is an apparent unification of Yukawa couplings $y_{t} \approx y_{b} \approx y_{\tau}$ and also a somewhat light Higgs sector has better chances of being probed at the LHC. Furthermore, for $\tan \beta \gg 1$, the offdiagonal mass mixing between the SM Higgs and the non-standard Higgs ${ }^{1}$ is suppressed as $\sin 2 \beta \sim 1 / \tan \beta$, which is very easy to see in the so-called "Higgs basis" [3]. This

\footnotetext{
${ }^{1}$ In the Higgs basis $h$ is the SM Higgs whose vacuum expectation value (vev) $\langle h\rangle=v=246 \mathrm{GeV}$, whereas $H$ is the non-standard Higgs that has vanishing vev $\langle H\rangle=0$.
} 
conclusion holds even with the inclusion of finite radiative corrections which are important to bring the lighter Higgs mass eigenstate to $125 \mathrm{GeV}$.

In this work we show that these constrained regions can be consistent with collider searches if there are additional decays for the heavy Higgs bosons which suppress the branching ratios of $H$ and $A$ to tau-leptons, $\operatorname{Br}(A, H \rightarrow \tau \bar{\tau})$. For that purpose we consider the possibility of having additional decays into pairs of sbottoms, stops and staus respectively. This has also been suggested in refs. [4-6] and studied in detail for electroweakinos in refs. $[7,8]$, where in the latter it was shown that SUSY decays into electroweakinos can be relevant for values of $5 \lesssim \tan \beta \lesssim 20$. We go beyond these studies by analysing the possible consequences on the destabilisation of the electroweak vacuum and flavour violating contributions, which impose an important constraint on the possible branching ratio to sfermions. We take into account the latest constraints on direct production of these SUSY particles. In particular, we exploit the left-right (LR) coupling of the heavy Higgs bosons to a pair of down-type sfermions which has a term proportional to $A_{f} \tan \beta$, that allows firstly to overcome the $\tan \beta$ enhancement of the usual dominant bottom-quark contribution to the total decay width and then to even possibly dominate the total decay for a sufficiently large value of the trilinear coupling $A_{f}$. In the case of stops we find it necessary to consider large values of the Higgs SUSY conserving mass $\mu$ in order to overcome the $\tan \beta$ enhancement.

We perform a numerical study and scan the parameter space, calculating the production cross-section for $H, A$ via gluon-fusion and in association with two bottom-quarks with SusHi 1.6.1 [9-19], ${ }^{2}$ and the decays and flavour observables with SARAH 4.11.0 [2224], SPheno 3.3.8 [25, 26], and flavio [27]. Finally we study possible stability issues with Vevacious 1.2.02 [28], which tend to constrain the maximum allowed values for $A_{f}$ and $\mu$. We find that indeed it is possible to partially recover some regions of the $m_{A}$-tan $\beta$ plane which seem to be disfavoured by current di-tau searches, enlarging the allowed large $\tan \beta$ regions in the MSSM.

The main theoretical considerations are discussed in section 2. Our results for light sbottoms are presented in section 3, for light staus in section 4, and for light stops in section 5. Finally, we conclude in section 6 .

\section{Analytical motivation}

We start with the tree-level coupling and decay rate expressions for the heavy Higgs bosons to fermions and sfermions. These expressions are well known and can be found for example in ref. [29]. We focus on the case of down-type fermions for which the couplings and decay rate take the form,

$$
\Gamma(\Phi \rightarrow d \bar{d})=N_{c} \frac{G_{F} M_{\Phi}}{4 \sqrt{2} \pi} m_{d}^{2} g_{\Phi \bar{d} d}^{2} \beta_{d}^{p}
$$

\footnotetext{
${ }^{2}$ See refs. [20, 21] for earlier calculations of QCD corrections to the decays of heavy Higgs bosons to quarks and squarks.
} 
where $\Phi=H, A, N_{c}$ is the colour factor, $p=3,1$ for CP-even or odd Higgs bosons, $\beta_{d}=\left(1-4 m_{d}^{2} / M_{\Phi}^{2}\right)^{1 / 2}$ and

$$
g_{H \bar{d} d}=\frac{\cos \alpha}{\cos \beta}, \quad g_{A \bar{d} d}=\tan \beta
$$

with $\alpha$ the usual Higgs mixing angle that relates the flavour to the mass eigenbasis. In fact, when $\tan \beta \gg 1, \alpha \rightarrow \beta-\pi / 2$ with the lightest Higgs CP-even mass eigenstate SM-like, implying that $g_{H \bar{d} d} \rightarrow \tan \beta$, so we see that both couplings are enhanced by $\tan \beta$. We should mention that couplings to up-type quarks on the other hand are suppressed in the same limit by $1 / \tan \beta$. From these expressions one can readily calculate $\operatorname{Br}(H, A \rightarrow \tau \bar{\tau})$ when only SM-particle decays are allowed since the total decay width is dominated by decays to bottom-quarks and find that $\operatorname{Br}(H, A \rightarrow \tau \bar{\tau}) \approx 0.1$, independently of $\tan \beta$ and $M_{\Phi}$. Thus this branching ratio is fixed. The dominant production mechanisms for $\Phi$, as mentioned before, are gluon fusion and production in association with bottom-quarks, with the latter dominating the production for very large values of $\tan \beta$. Given that $g_{\Phi \bar{d} d}$ enters linearly in both production diagrams, we clearly see that there will be a dependence of the form $\sigma_{H, A} \times \operatorname{Br}(H, A \rightarrow \tau \bar{\tau}) \propto \tan ^{2} \beta$, where $\sigma_{H, A}$ represents both production mechanisms, from which we understand how the constraints for large values of $\tan \beta$ come about.

The couplings and decay rates for sfermions take the form,

$$
\Gamma\left(\Phi \rightarrow \tilde{f}_{i} \tilde{f}_{j}\right)=N_{c} \frac{G_{F}}{2 \sqrt{2} \pi M_{\Phi}} \lambda_{\tilde{f}_{i} \tilde{f}_{j} \Phi}^{1 / 2} g_{\Phi \tilde{f}_{i} \tilde{f}_{j}}^{2}
$$

with $\tilde{f}_{i}, i=1,2$ the sfermion mass eigenstates and $\lambda_{\tilde{f}_{i} \tilde{f}_{j} \Phi}$ is the well known Kallen lambdafunction which appears in the kinematics of a two-body decay,

$$
\lambda_{i j k}=\left(1-\frac{M_{i}^{2}}{M_{k}^{2}}-\frac{M_{j}^{2}}{M_{k}^{2}}\right)^{2}-4 \frac{M_{i}^{2} M_{j}^{2}}{M_{k}^{4}} .
$$

Notice that contrary to the case of decay to fermions which grows with $M_{\Phi}$, decays to sfermions are suppress by $1 / M_{\Phi}$. The couplings $g_{\Phi \tilde{f}_{i} \tilde{f}_{j}}$ are combinations of chiral-couplings,

$$
g_{\Phi \tilde{f}_{i} \tilde{f}_{j}}=\sum_{\alpha, \beta=L, R} T_{i j \alpha \beta} g_{\Phi \tilde{f}_{\alpha} \tilde{f}_{\beta}}
$$

The couplings with the same chirality have terms proportional to SM fermions or gauge boson masses and thus are not efficient in enhancing these couplings. Interestingly, the mixed-chirality couplings take the form,

$$
\begin{array}{rlrl}
g_{A \tilde{d}_{L} \tilde{d}_{R}} & =-\frac{1}{2} m_{d}\left[\mu+A_{d} \tan \beta\right], & g_{H \tilde{d}_{L} \tilde{d}_{R}} & =-\frac{1}{2} m_{d}\left[\frac{\sin \alpha}{\cos \beta} \mu+A_{d} \frac{\cos \alpha}{\cos \beta}\right] \\
g_{A \tilde{u}_{L} \tilde{u}_{R}}=-\frac{1}{2} m_{u}\left[\mu-\frac{1}{\tan \beta} A_{u}\right], & g_{H \tilde{u}_{L} \tilde{u}_{R}}=-\frac{1}{2} m_{u}\left[\frac{\cos \alpha}{\sin \beta} \mu+A_{u} \frac{\sin \alpha}{\sin \beta}\right]
\end{array}
$$

which depend on the SUSY breaking trilinear couplings $A_{f}$ and SUSY conserving mass $\mu$. Thus in the large $\tan \beta$ regions we see that there will be terms enhanced by $\tan \beta$ proportional to $A_{d}$ which can be used to increase the couplings to down-type sfermions. In 
the case of couplings to up-type sfermions, there is only at most a term independent of $\tan \beta$ growing with $\mu$ which can be used to increase the coupling. ${ }^{3}$ The factor $T_{i j \alpha \beta}$ takes into account the chiral mixing in the mass basis and in order to maximise the couplings we should be close to maximal mixing $\sin \theta_{f} \approx \cos \theta_{f} \approx 1 / \sqrt{2}$, with $\theta_{f}$ the mixing angle. We take also into account important loop-level contributions which modify the relation between down-type Yukawas and running masses,

$$
y_{b}=\frac{m_{b}}{v \cos \beta\left(1+\Delta_{b}\right)}, \quad y_{\tau}=\frac{m_{\tau}}{v \cos \beta\left(1+\Delta_{\tau}\right)}
$$

where $\Delta_{b}$ is dominated by sbottom-gluino and stop-chargino loop, whereas $\Delta_{\tau}$ is dominated by stau-neutralino and sneutrino-chargino loop, and both can be sizeable in the large $\tan \beta$ regime.

Given that we want the contribution from the L-R coupling to be maximal in order to enhance the decays into SUSY particles, we must choose the soft breaking masses to be roughly of the same order in particular for the sbottom and stau sectors due to their smaller Yukawa couplings. This implies that we expect $m_{\tilde{b}_{2}} \gtrsim m_{\tilde{b}_{1}}$ and $m_{\tilde{\tau}_{2}} \gtrsim m_{\tilde{\tau}_{1}}$, which we find in our numerical studies. For stops the story is different given their important loop contribution to the effective Higgs potential which pushes the lightest Higgs mass to $125 \mathrm{GeV}$. In this case one must choose one of the soft breaking masses $\left(m_{U_{3}}\right.$ or $\left.m_{Q_{3}}\right)$ to be of the same magnitude as $A_{t} \simeq 2 \mathrm{TeV}$, pushing the heavier stop in the few $\mathrm{TeV}$ region. However, since we want to have the heavy Higgs bosons decay to stops in the first place, we need the lighter stop to remain light enough to kinematically allow for such decays. Thus in this case the spectrum is more split $\left(m_{\tilde{t}_{1}} \ll m_{\tilde{t}_{2}}\right.$ and $\left.m_{\tilde{t}_{1}} \lesssim m_{\Phi} / 2\right)$ than for sbottoms and staus and though the mixing is not maximal we can still have stops contributing to the total decay width enough to suppress $\operatorname{Br}(\Phi \rightarrow \tau \bar{\tau})$.

Large values of $A_{b}, A_{\tau}$ and $\mu$ are constrained by colour and electromagnetic charge breaking since they provide cubic terms in the scalar potential that tend to destabilise the neutral electroweak symmetry breaking vacuum [30]. We will see in the next section that this puts strong constraints on the allowed values for $A_{b}, A_{\tau}$ and $\mu$. There are flavour violating processes which are enhanced at large $\tan \beta$, in particular B-meson decays (See e.g. [31]). We will also comment on this in the next sections. We perform a numerical scan for each of the three discussed possibilities.

\section{Light sbottoms}

We describe the parameter space and the codes used in the numerical scan for light sbottom quarks in the next section, before discussing our results in section 3.2.

\subsection{Numerical scan}

In order to study the feasibility to enlarge regions of large values of $\tan \beta$ currently constrained by $H, A \rightarrow \tau \bar{\tau}$ searches, we do a numerical simulation of the productions of $H$ and $A$ via gluon fusion and in association with bottom-quark pairs using SusHi 1.6.1 [9-19],

\footnotetext{
${ }^{3}$ The trilinear interaction for up-type sfermions is suppressed by $1 / \tan \beta$ as shown in eq. (2.6).
} 
a Fortran code which can calculate these production cross sections in the MSSM. In the case of gluon fusion, it takes into account $N L O$ QCD contributions from the third family of quarks and squarks, $N^{3} L O$ corrections due to top-quarks, approximate NNLO corrections due to top squarks and electroweak effects. Very much relevant for large values of $\tan \beta$ for the down-type sector and it particular for the third family Yukawa couplings, it resums higher order $\tan \beta$-enhanced sbottom contributions. The supersymmetric particle spectrum, as well as cross-sections and decays for SUSY particles, are calculated using SARAH 4.11.0 [22-24] and SPheno 3.3.8 [25, 26], in particular the SPheno version generated from the MSSM model file in SARAH. We subsequently calculate flavour observables with flavio [27], which takes the Wilson coefficients calculated by FlavorKit [24] as input, and the Higgs production cross sections at the LHC with SusHi 1.6.1 [9-19] for both CP even Higgs bosons using the MMHT 2014 [32] parton distribution functions set via LHAPDF 6.1.6 [33]. Stability of the electroweak vacuum and possible charge/colour breaking minima are investigated using Vevacious 1.2.02 [28], which relies on CosmoTransitions [34] and HOM4PS2 [35]. Due to the lack of SUSY signals so far at the LHC, we decide to consider a natural spectrum, pushing $1^{\text {st }}$ and $2^{\text {nd }}$-generation sparticles, as well as gluinos and Winos in the multi- $\mathrm{TeV}$ range:

$$
m_{\tilde{e}_{j}}=m_{\tilde{L}_{j}}=m_{\tilde{u}_{i}}=m_{\tilde{d}_{i}}=m_{\tilde{Q}_{i}}=M_{2}=M_{3}=2.2 \mathrm{TeV}
$$

with vanishing $A$-terms. For $3^{\text {rd }}$-generation sparticles, depending on how we want to suppress the branching ratio $\operatorname{Br}(H, A \rightarrow \tau \bar{\tau})$, we keep either sbottoms, staus or stops light ${ }^{4}$ to allow for heavy Higgs SUSY decays to be kinematically accessible. Since $|\mu|, M_{1} \ll$ $M_{2}, M_{3}, m_{\tilde{f}_{1,2}}$, the other possible light sparticles in the spectrum are the first three lighter neutralinos $\tilde{\chi}_{1}^{0}, \tilde{\chi}_{2}^{0}, \tilde{\chi}_{3}^{0}$ and the light chargino $\tilde{\chi}_{1}^{ \pm}$. In the scan with light sbottoms, we fixed

$$
M_{1}=200 \mathrm{GeV} \quad m_{\tilde{u}_{3}}=2845 \mathrm{GeV},
$$

and varied the remaining parameters, $\tan \beta, \mu, B_{\mu}, m_{\tilde{Q}_{3}}, m_{\tilde{d}_{3}}$, and $A_{t}$

$$
\begin{array}{rlrl}
\tan \beta & \in[25,60] & m_{\tilde{Q}_{3}} & \in[300,800] \mathrm{GeV} \\
\mu & m_{\tilde{d}_{3}} \in[300,800] \mathrm{GeV} \\
m_{A}(\text { tree }) & \in[500,1600] \mathrm{GeV} & A_{t} & = \pm m_{\tilde{u}_{3}} .
\end{array}
$$

For all points with $m_{\tilde{b}_{1}} \geq 300 \mathrm{GeV}$, which are close to the experimental exclusion limit of the $H \rightarrow \tau \bar{\tau}$ searches and for which the decay $H \rightarrow \tilde{b} \tilde{b}^{*}$ is kinematically accessible, we increased $\left|A_{b}\right|$, both for positive and negative $A_{b}$, and used a fixed-point iteration to determine the largest possible value, for which the electroweak vacuum is either stable or sufficiently longlived on cosmological scales. Finally, we increased $M_{1}$ to enlarge the parameter space by further suppressing the limits from the direct sbottom pair production searches.

We impose that the lightest Higgs particle in the spectrum, which is associated with the scalar resonance discovered at the LHC, satisfies the measurement of the Higgs mass $125 \pm$ $3 \mathrm{GeV}$ taking the theory error into account and the latest signal strengths measurements

\footnotetext{
${ }^{4}$ In order to obtain large enough radiative corrections to increase the light Higgs mass to $\sim 125 \mathrm{GeV}$, one tends to need large values of $A_{t}$ which can lead to a light stop in the spectrum.
} 
by ATLAS and CMS at the $2 \sigma$ level (table 16 in ref. [36]), in the different relevant channels: $b \bar{b}, W W^{*}, Z Z^{*}, \tau \bar{\tau}$ and $\gamma \gamma$. We discarded all data points, which have a sbottom quark mass below $300 \mathrm{GeV}$ to satisfy mono-jet searches at $3.2 \mathrm{fb}^{-1}$ [37] and directly use the latest $13 \mathrm{TeV}$ CMS direct sbottom [38] and stop [39] pair production searches with a luminosity $\mathcal{L}=36.1 \mathrm{fb}^{-1}$ by imposing the limit extracted from the provided root files, where we use the QCD squark pair production cross section reported in ref. [40].

For the main object of our study, the heavy Higgs bosons $H, A$, we require that both the productions in association with bottom-quarks and via gluon fusion, with subsequent decay into tau pairs, $\sigma_{b b H} \times \operatorname{Br}(H \rightarrow \tau \bar{\tau})$ and $\sigma_{g g H} \times \operatorname{Br}(H \rightarrow \tau \bar{\tau})$, satisfy the bounds from both ATLAS [2] and CMS [1] studies at $13 \mathrm{TeV}$ and $13.3 \mathrm{fb}^{-1}$ and $12.9 \mathrm{fb}^{-1}$, respectively, though due to the large values of $\tan \beta$ we are interested in, the production in association with bottom-quark pairs places stronger constraints.

We make a few comments with respect to flavour observables and constraints. We are able to satisfy all flavour observable constraints $\left(B_{s} \rightarrow \mu^{+} \mu^{-}\right.$[41], $B \rightarrow \tau \nu$ [42], etc) at the $2 \sigma$ level, except for $B \rightarrow X_{s} \gamma$ [42], for which the stop-chargino loop contribution can be significant, whereas the charged Higgs contributions seems to be subdominant. Within the Minimal Flavour violation (MFV) paradigm, a study done in ref. [31] shows that for $A_{t}>0, \mu \gtrsim 800 \mathrm{GeV}$ or $M_{Q_{3}} \gtrsim 1.3 \mathrm{TeV}$ are necessary to satisfy the latest measurements. For $A_{t}<0$, constraints are much stronger and always require $M_{Q_{3}} \gtrsim 1.5 \mathrm{TeV}$. Since we want to have a light enough sbottom for the heavy Higgs bosons to decay, this implies that the only possibility would be to have $A_{t}>0$ and $\mu \gtrsim 800 \mathrm{GeV}$, which would not affect the main conclusions of this work. However, recall that this is all within the MFV paradigm. Beyond the MFV paradigm, there are new ways to suppress the contribution of the stopchargino loop, in particular possible additional diagrams involving gluinos and sbottomstrange mixing, which may be able to cancel the chargino-stop contributions [43, 44]. Thus we do not impose in our results the constraint from $B \rightarrow X_{s} \gamma$ due to the caveats just discussed.

\section{$3.2 \quad$ Results}

Having taken in consideration all these constraints, we show our results in figures 1,2 and 3. Grey points are excluded by $A, H \rightarrow \tau \bar{\tau}$ searches, blue points have $A_{b}=0$ such that decays into sbottoms are negligible, orange and red points have $A_{b} \neq 0$ and thus a nonnegligible decay into sbottoms. Orange points feature either a fully stable or a metastable electroweak vacuum. In the plot on the left-hand side of figure 1, we furthermore indicate data points with a fully stable electroweak vacuum and $A_{b} \neq 0$ in dark orange. The black and red points connected by dashed/dotted lines are two particular examples where we only vary $A_{b}$ while keeping all other parameters fixed, in order to show how we move in the particular planes shown. The big black dots represent the points with $A_{b}=0$ and the purple arrow points in the direction of increasing $A_{b}$.

In figure 1 on the left, we plot $\operatorname{Br}(H \rightarrow \tau \bar{\tau})$ vs $A_{b}$. We immediately see from the red and orange points that as $A_{b}$ grows in magnitude, we are able to suppress the $\operatorname{Br}(H \rightarrow \tau \bar{\tau})$ via the additional sbottom decays by factors of order a half or slightly smaller. There are however points which have a large $A_{b}$ but nonetheless a large $\operatorname{Br}(H \rightarrow \tau \bar{\tau})$, which 

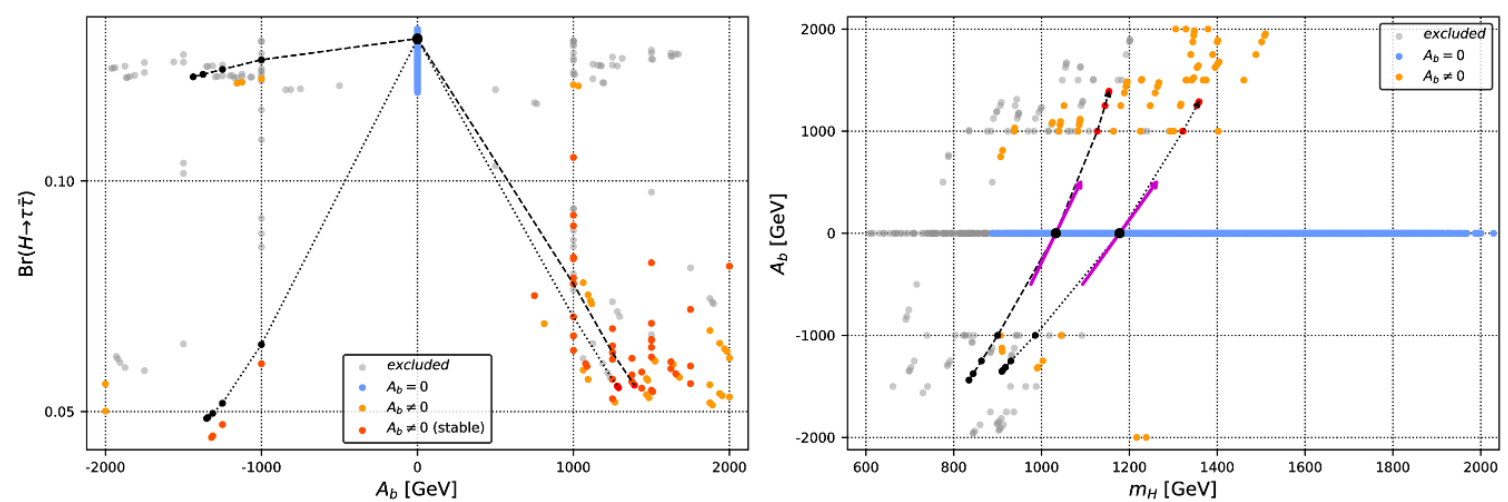

Figure 1. $\operatorname{Br}(H \rightarrow \tau \bar{\tau})$ vs $A_{b}[\mathrm{GeV}]$ and $A_{b}[\mathrm{GeV}]$ vs $m_{H}[\mathrm{GeV}]$. Grey points are excluded by $A, H \rightarrow \tau \bar{\tau}$ searches, blue points have $A_{b}=0$ such that decays into sbottoms are negligible, orange and red points have $A_{b} \neq 0$ and thus a non-negligible decay into sbottoms. All orange points have either a fully stable or metastable electroweak vacuum. In the left-hand figure, we distinguish between the stable and metastable vacuum. Dark orange points in the left-hand figure feature a fully stable electroweak vacuum and lighter orange points have a metastable electroweak vacuum. Black and red points on top of the dashed and dotted black lines are two particular examples where we only vary $A_{b}$ while keeping all other parameters fixed, in order to show how we move in the particular planes shown. The purple arrow indicates the direction of increasing $A_{b}$. Direct sbottom searches as well as light Higgs bound constraints are satisfied by all non-excluded points.

implies that these points do not correspond to maximal mixing between the left and right handed sbottoms. Note as well that we find both metastable and fully stable vacua for $\left|A_{b}\right| \lesssim 2 \mathrm{TeV}$. In the two examples shown in this figure we leave everything fixed except $A_{b}$ and one can see that as $A_{b}$ increases in magnitude one is able to suppress via decays into sbottoms the $\operatorname{Br}(H \rightarrow \tau \bar{\tau})$. However, for the two examples the suppression is not sufficient enough to avoid the LHC constraints from $H, A \rightarrow \tau \bar{\tau}$ for one the branches $\left(A_{b}<0\right)$. Comparing the location of most of the red and orange points against the grey points, it is clear that a suppression in $\operatorname{Br}(H \rightarrow \tau \bar{\tau})$ is what allows them to evade the di-tau constraints. There are, however, some stragglers for which $\operatorname{Br}(H \rightarrow \tau \bar{\tau}) \gtrsim 0.1$ and are able however to evade the constraints. These points correspond to large $m_{H}$ such that the constraints from di-taus ameliorate. In figure 1 on the right on the other hand, we show the influence of $A_{b}$ on $m_{H}$. This is clearly seen in the two example black dashed/dotted lines in this figure, where as we move $A_{b}$ keeping all other parameters fixed, we see that $m_{H}$ can either decrease or increase by several GeV's, even $\Delta m_{H} \sim 100 \mathrm{GeV}$. This is coming from the radiative sbottom corrections to the effective Higgs potential, that as we see for large values of $\tan \beta$ can be quite relevant [45]. In the two examples $m_{H}$ increases with increasing $A_{b}$, but the opposite behaviour, where $m_{H}$ decreases with increasing $A_{b}$, also occurs for some points in the numerical scan.

This last analysis helps us to partially understand figure 2. In the figure on the right, we show the main constraining production cross section $\sigma_{b b H}$ as a function of $m_{H}$. We see that as $m_{H}$ increases there is a clear reduction in the cross section as expected. Furthermore, we see this explicitly in the two examples represented once again by the dashed black lines. 

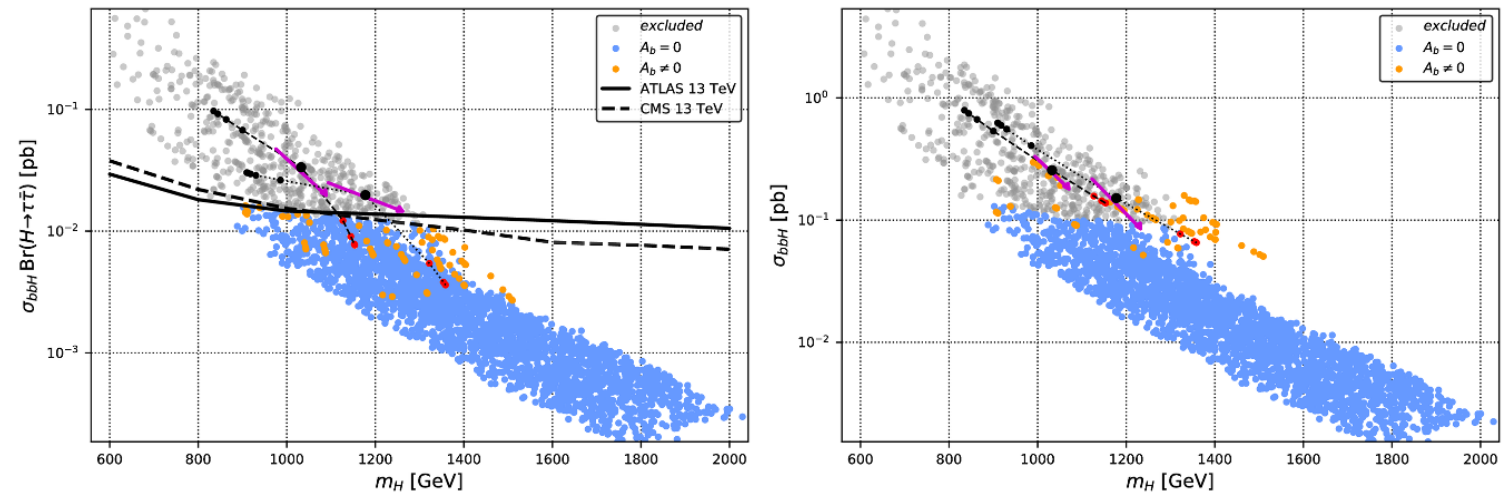

Figure 2. $\sigma_{b b H} \times \operatorname{Br}(H \rightarrow \tau \bar{\tau})[\mathrm{pb}]$ vs $m_{H}[\mathrm{GeV}]$ and $\sigma_{b b H}[\mathrm{pb}]$ vs $m_{H}[\mathrm{GeV}]$. Same colour coding for points as in figure 1. Solid and dashed horizontal lines in the figure on the left represent the constraints from the latest ATLAS and CMS $13 \mathrm{TeV} A, H \rightarrow \tau \bar{\tau}$ searches at $13.3 \mathrm{fb}^{-1}$, respectively. We still show the same two example points where we only vary $A_{b}$.

Here we see the effect of $A_{b}$ shifting $m_{H}$ and reducing or increasing the cross section. On the other hand, on the left of figure 2 , we show $\sigma_{b b H} \times \operatorname{Br}(H \rightarrow \tau \bar{\tau})$ vs $m_{H}$. We also display the constraints from the latest ATLAS [2] and CMS [1] $A, H \rightarrow \tau \bar{\tau}$ searches at $13 \mathrm{TeV}$, respectively, represented by the solid and dashed nearly horizontal lines in the figure, showing clearly that the grey points are excluded by these searches. Now we see in the two examples that we have chosen, that the initial points with $A_{b}=0$ are right at the border of exclusion and as we vary $A_{b}$, we either move into the non-excluded area by two effects: a decrease in the production cross section due to a larger $m_{H}$ and a decrease in the $\operatorname{Br}(H \rightarrow \tau \bar{\tau})$ due to di-sbottom decays. Indeed, we see that the line of the two example becomes steeper as we move in the non-excluded area. We can however, also move deeper into the excluded area as depicted by the two examples, by a decrease in $m_{H}$ (which leads to an increase in the production cross section) and an insufficient suppression of the branching ratio $\operatorname{Br}(H \rightarrow \tau \bar{\tau})$.

Finally in figure 3 , we show $\tan \beta$ vs $m_{H}$. The grey envelope area of the excluded points indicates the excluded region. We see something very interesting happening. Most of the orange points lie within the grey envelope, implying that without the additional suppression due to the decays into sbottom pairs from the heavy Higgs, they would have been ruled out by the current $H, A \rightarrow \tau \bar{\tau}$ searches. The blue points that lie also within the grey envelope have a maximal $\operatorname{Br}(H \rightarrow \tau \bar{\tau}) \sim 0.1$ as shown in figure 1 on the left, but as seen from figure 2 on the right, they have a somewhat suppressed production cross section with respect to the grey points. This is most likely due to a suppressed coupling from radiative corrections for these points as shown in eq. (2.7). This effect has been discussed for decays to stau pairs in ref. [5].

Focusing on the two example lines, we see that as we change $A_{b}$, we move horizontally in the plane $m_{H}-\tan \beta$ due to the change in $m_{H}$ as $A_{b}$ varies. In both examples we see that starting from the points with $A_{b}=0$ which are represented by the slightly larger black dots, as $m_{H}$ becomes larger we are able to obtain viable points (red points) which 


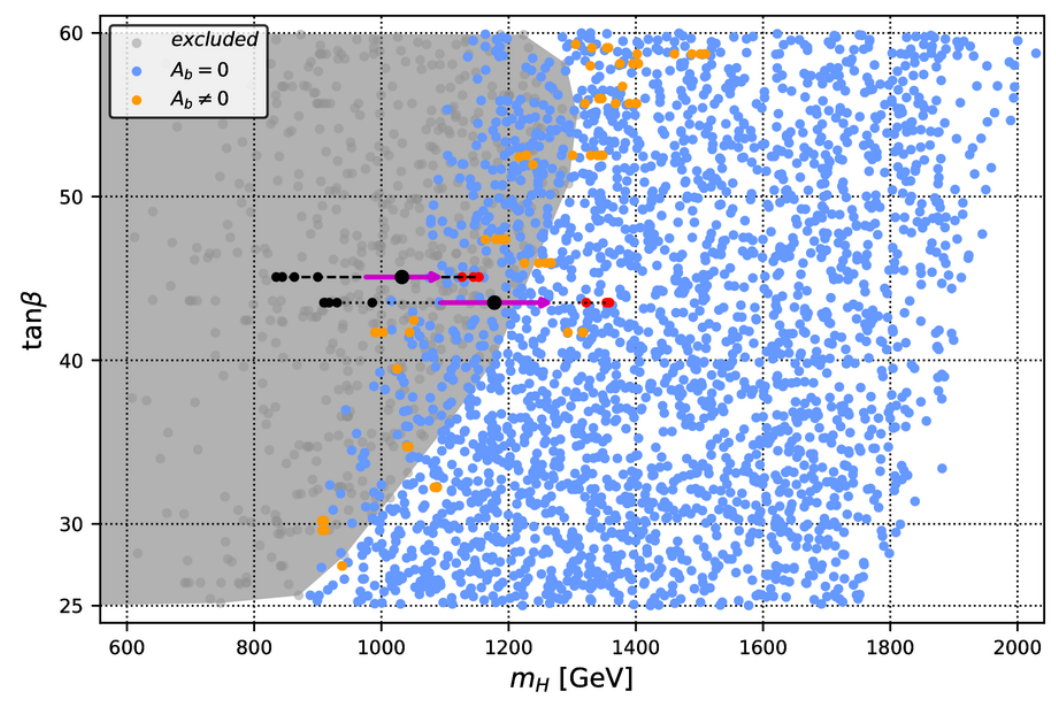

Figure 3. Tan $\beta$ vs $m_{H}$ [GeV]. Same colour coding for points as in figure 1. Dark grey region is the envelope of the excluded points. The most interesting points are the orange points that land in the dark grey envelope. We still show the same two example points where we only vary $A_{b}$.

are within the grey envelope. On the other hand, to the other side of the big black dot, we move to smaller $m_{H}$ but are further excluded. These two behaviours can be understood by looking how in the two examples $m_{H}$ and $\operatorname{Br}(H \rightarrow \tau \bar{\tau})$ depend on $A_{b}$ as shown in figure 1 . Although $\operatorname{Br}(H \rightarrow \tau \bar{\tau})$ diminishes for increasing $\left|A_{b}\right|$ in both examples, the decrease in $\operatorname{Br}(H \rightarrow \tau \bar{\tau})$ for negative $A_{b}$ is compensated by the increased production cross section for a lighter $H$. For positive $A_{b}, m_{H}$ increases with increasing $A_{b}$ and thus the production cross section is reduced in addition to the suppression of the branching ratio $\operatorname{Br}(H \rightarrow \tau \bar{\tau})$.

\section{Light staus}

The numerical scan for light staus is very similar to the one for light sbottoms. We discuss any differences to the scan for sbottoms in the next subsection and our results in section 4.2.

\subsection{Numerical scan}

We decouple winos, squarks, and the first two generations of sleptons

$$
m_{\tilde{Q}_{j}}=m_{\tilde{u}_{j}}=m_{\tilde{d}_{j}}=m_{\tilde{L}_{i}}=m_{\tilde{e}_{i}}=M_{2}=M_{3}=2.2 \mathrm{TeV}
$$

and similarly fix the bino mass and the right-handed stop mass to a large enough loop correction to the Higgs mass

$$
M_{1}=100 \mathrm{GeV} \quad m_{\tilde{u}_{3}}=2845 \mathrm{GeV} .
$$

The other parameters are varied

$$
\begin{aligned}
\tan \beta & \in[25,60] & m_{\tilde{L}_{3}} & \in[150,800] \mathrm{GeV} \\
\mu & \in \pm[200,400] \mathrm{GeV} & m_{\tilde{e}_{3}} & \in[150,800] \mathrm{GeV} \\
m_{A}(\text { tree }) & \in[500,1600] \mathrm{GeV} & A_{t} & = \pm m_{\tilde{u}_{3}} .
\end{aligned}
$$



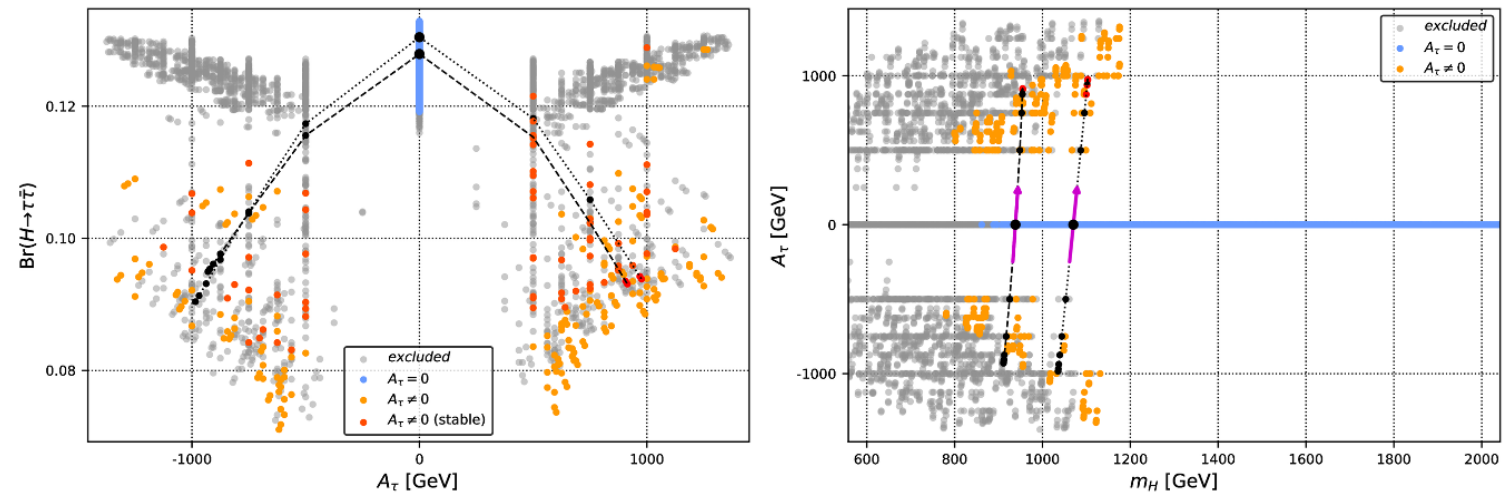

Figure 4. $\operatorname{Br}(H \rightarrow \tau \bar{\tau})$ vs $A_{\tau}[\mathrm{GeV}]$ and $A_{\tau}[\mathrm{GeV}]$ vs $m_{H}[\mathrm{GeV}]$. Same colour coding for points as in figure 1.
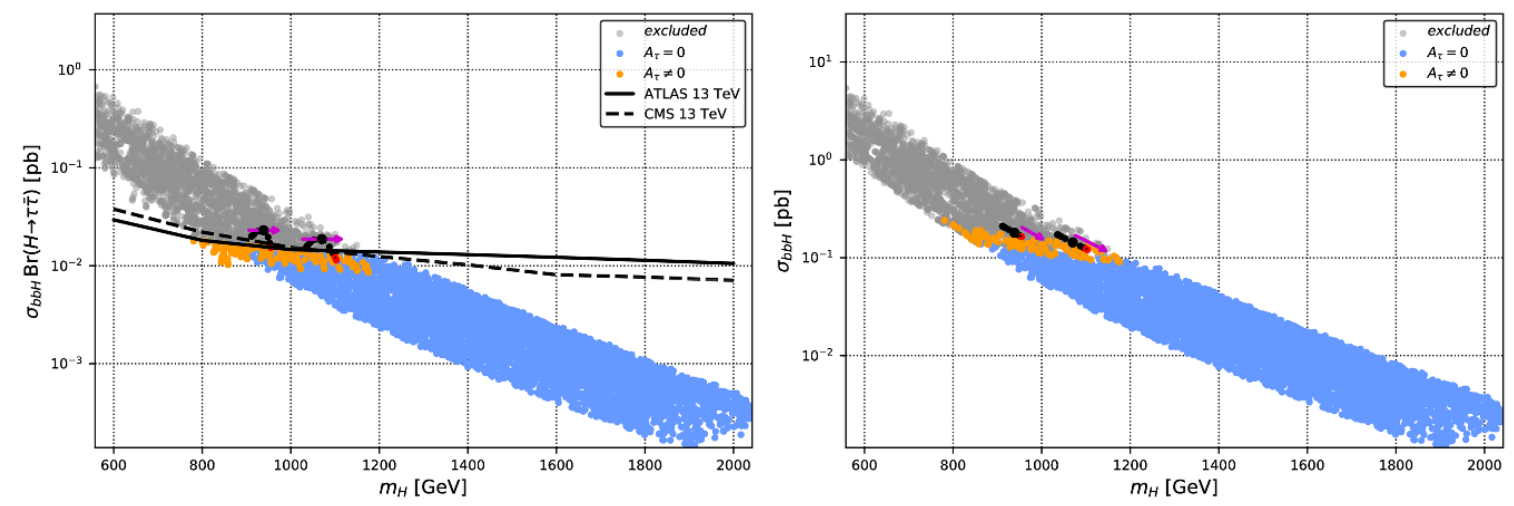

Figure 5. $\sigma_{b b H} \times \operatorname{Br}(H \rightarrow \tau \bar{\tau})[\mathrm{pb}]$ vs $m_{H}[\mathrm{GeV}]$ and $\sigma_{b b H}[\mathrm{pb}]$ vs $m_{H}[\mathrm{GeV}]$. Same colour coding for points as in figure 1 .

Initially we keep $A_{\tau}=0$ fixed and in a second step, we increase $\left|A_{\tau}\right|$, and use a fixedpoint iteration to determine the largest possible value with a stable or long-lived electroweak vacuum.

Direct stop and sbottom pair production searches are automatically satisfied and we conservatively require $m_{\tilde{\tau}} \geq 100 \mathrm{GeV}$ to satisfy the current limits on the $\tilde{\tau}$ mass [42] and that the lightest supersymmetric particle is a neutralino. All flavour constraints, and in particular, $B_{s} \rightarrow \mu^{+} \mu^{-}$[41], $B \rightarrow \tau \nu$ [42], $B \rightarrow X_{s} \gamma$ [42], are satisfied at the $2 \sigma$ level. Similarly to the scan with light sbottoms, we impose the Higgs signal strength measurements at $2 \sigma$ as well as the Higgs mass measurement.

\subsection{Results}

The colour coding in figures 4 to 6 is the same as for the sbottom case, with the obvious replacements.

In figure 4 on the left, we see that we can still suppress the $\operatorname{Br}(H \rightarrow \tau \bar{\tau})$ via decays into stau pairs. However, in comparison with the decays into sbottoms, the suppression is less effective which may be related to the number of colour that enters in the sbottom 


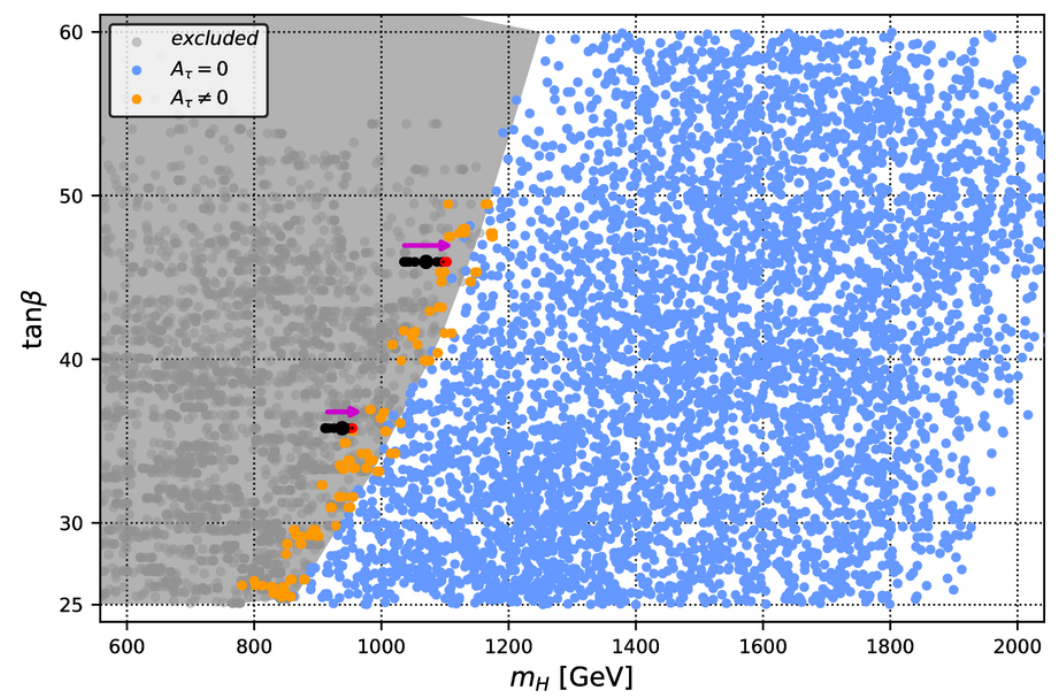

Figure 6. Tan $\beta$ vs $m_{H}[\mathrm{GeV}]$. Same colour coding for points as in figure 1.

decay case, as well as the larger Yukawa coupling of the bottom-quark with respect to the tau-quark, see eq. (2.3). Furthermore, the smallest values of $\operatorname{Br}(H \rightarrow \tau \bar{\tau})$ which are stable occur for somewhat small values of $A_{\tau},\left|A_{\tau}\right| \sim 600 \mathrm{GeV}$. We have checked that larger values of $A_{\tau}$ would lead to stronger suppressions of $\operatorname{Br}(H \rightarrow \tau \bar{\tau})$, however they are excluded by vacuum stability constraints.

In figure 4 on the right, contrary to the sbottom case, the dependence of $m_{H}$ on $A_{\tau}$ is much milder once again due to the smaller Yukawa and the lack of colour for the stau case. Note that the orange points for which $A_{\tau} \neq 0$ and which are stable, start at $m_{H} \gtrsim 800 \mathrm{GeV}$. This can be understood by looking at figure 5 on the left, where due to our scanning procedure which starts with points that are barely ruled out by the $H \rightarrow \tau \bar{\tau}$ searches and considers $\tan \beta>25$, the lightest mass $m_{H}$ we can obtain which is barely ruled out is $m_{H} \approx 800 \mathrm{GeV}$. If we had chosen a lower value of $\tan \beta$, we could have observed the stau effect for smaller values of $m_{H}$. Nonetheless, by looking at the two example points and also at the "width" of the orange region, we conclude that the effect of staus is much less significant in allowing a larger parameter region than that of sbottoms. This can also be seen in figure 5 on the right.

Finally in figure 6 , we translate the results to the $m_{H}-\tan \beta$ plane. We see that indeed one can get most of the orange points in the would-be excluded region, delimited by the grey envelope. Again we see that the depth of the orange points in the grey envelope is much thinner compared to the sbottom case of figure 3 .

There has been an analytical study of the stau case in ref. [5]. It showed that, at the time, a suppression of up to $20 \%$ of $\sigma_{b b H} \times \operatorname{Br}(H \rightarrow \tau \bar{\tau})$ with respect to the case without SUSY decays was achievable for values of $A_{\tau} \sim 1.3 \mathrm{TeV}$. We find slightly better results in our numerical study, as can been seen in figure 5 on the left, where we estimate up to $50 \%$ suppression for $\sigma_{b b H} \times \operatorname{Br}(H \rightarrow \tau \bar{\tau})$ with respect to the case with no SUSY decays. Notice also that values of $A_{\tau} \sim 1.3 \mathrm{TeV}$ are on the borderline of metastability, as shown 
in figure 4 on the left and that though an analytical study for the stau case is consistent, a similar one for the sbottom case is not straightforward due to the large dependence of $m_{H}$ on $A_{b}$.

\section{$5 \quad$ Light stops}

In the case of stops, given that we want only stops and not sbottoms to be light and that to obtain a mass for the lightest Higgs $h$ of $m_{h} \approx 125 \mathrm{GeV}$, which implies $A_{t} \sim m_{Q_{3}} \sim 2 \mathrm{TeV}$, we have in the end that one stop is light (mostly right-handed) while the other stop is much heavier (mostly left-handed). We also consider values of $\tan \beta \in[25,60]$. As mentioned in section 2, the way to increase the branching ratio of $\mathrm{H}$ into stops is by increasing the value of $\mu$. However, there are large radiative corrections to the heavy Higgs mass $m_{H}$ which are much stronger than in the case of $A_{b}$ or $A_{\tau}$ for the sbottom and stau cases. Thus the scanning procedure of leaving everything fixed except $\mu$ is much less efficient and we are only able to retrieve stable points for $m_{H}>2.6 \mathrm{TeV}$ and $\mu>2.4 \mathrm{TeV}$, with very small branching ratio into stops. Vacuum stability is only an issue for the very largest values of $\mu \gtrsim 4.5 \mathrm{TeV}$. Performing a random scan we were able to see the effect of stops reducing the $\operatorname{Br}(H \rightarrow \tau \bar{\tau})$ via a $\operatorname{Br}\left(H \rightarrow \tilde{t}_{1} \tilde{t}_{1}^{*}\right) \lesssim 0.4$. Their effect seems to start at $m_{H} \gtrsim 1 \mathrm{TeV}$ and extend up to $m_{H} \approx 2.2 \mathrm{TeV}$ for values of $\mu \in[1.2,3.5] \mathrm{TeV}$. The problem however in this case by performing a random scan is that we loose the guide from the two sets of example points which we showed for the sbottom and stau cases, respectively. Thus we decided to only comment briefly on this possibility in reducing $\operatorname{Br}(H \rightarrow \tau \bar{\tau})$.

\section{Conclusion}

Searches for heavy Higgs bosons decaying to a pair of $\tau$ leptons severely constrain the parameter space of the MSSM for large $\tan \beta$. We demonstrate three possible ways how to alleviate the constraints by new decay channels into third-generation sfermion pairs. For large $\tan \beta$, the coupling of the heavy Higgs to the sbottoms and staus proportional to $\tan \beta$ can be further enhanced by a large value of the trilinear couplings $A_{b}$ and $A_{\tau}$, respectively, while the coupling to stops has a $\tan \beta$ independent part which can be enhanced by the SUSY conserving Higgs mass $\mu$. The maximum size of the trilinear couplings $A_{f}$ and $\mu$, however, is constrained by the stability of the electroweak vacuum.

Our numerical scan shows that light sbottoms have the greatest potential to alleviate the constraints from heavy Higgs searches. After imposing vacuum stability, $\left|A_{b}\right|$ can take values up to $2 \mathrm{TeV}$ and leads to a reduction of the branching ratio $\operatorname{Br}(H \rightarrow \tau \bar{\tau})$ by more than a factor two down to $\operatorname{Br}(H \rightarrow \tau \bar{\tau}) \lesssim 0.05$, which enlarges the available parameter space. Similarly, for light staus we find values of $\left|A_{\tau}\right| \sim 1.3 \mathrm{TeV}$ with a reduced branching ratio $\operatorname{Br}(H \rightarrow \tau \bar{\tau}) \sim 0.07$, which allows to slightly enlarge the allowed region of parameter space. Finally, light stops allow very large values of $|\mu|$ close to $5 \mathrm{TeV}$. However, radiative corrections to the heavy Higgs mass $m_{H}$ are large and substantially increase it. It is still possible to observe the effect of a reduced branching ratio $\operatorname{Br}(H \rightarrow \tau \bar{\tau})$ via an increased 
branching ratio for the decay into light stops $\operatorname{Br}\left(H \rightarrow \tilde{t}_{1} \tilde{t}_{1}^{*}\right) \lesssim 0.4$, but a detailed discussion would require to fix the heavy Higgs mass $m_{H}$ as much as possible when increasing $|\mu|$.

Although future searches for heavy Higgs bosons decaying to $\tau$-pairs may tighten the constraints on the MSSM parameter space and eventually exclude the orange points in the figures, our main conclusion, that new decays to light third-generation fermions will alleviate the constraints from heavy Higgs search, holds irrespectively. This scenario can be tested by improving the reach of the searches for light third generation sfermions.

\section{Acknowledgments}

We thank Carlos Wagner for useful discussions. This work has been supported by the European Research Council (ERC) Advanced Grant Higgs@LHC. This work was supported in part by the Australian Research Council. We acknowledge the use of matplotlib 2.0.1 [46, 47] and Julia 0.5.2 [48, 49]. This research was supported by use of the Nectar Research Cloud, a collaborative Australian research platform supported by the National Collaborative Research Infrastructure Strategy (NCRIS).

Open Access. This article is distributed under the terms of the Creative Commons Attribution License (CC-BY 4.0), which permits any use, distribution and reproduction in any medium, provided the original author(s) and source are credited.

\section{References}

[1] CMS collaboration, Search for a neutral MSSM Higgs boson decaying into $\tau \tau$ with $12.9 \mathrm{fb}^{-1}$ of data at $\sqrt{s}=13 \mathrm{TeV}$, CMS-PAS-HIG-16-037 [INSPIRE].

[2] ATLAS collaboration, Search for Minimal Supersymmetric Standard Model Higgs Bosons $H / A$ in the $\tau \tau$ final state in up to $13.3 \mathrm{fb}^{-1}$ of $p p$ collisions at $\sqrt{s}=13$ TeV with the ATLAS Detector, ATLAS-CONF-2016-085 [INSPIRE].

[3] M. Carena, I. Low, N.R. Shah and C.E.M. Wagner, Impersonating the Standard Model Higgs Boson: Alignment without Decoupling, JHEP 04 (2014) 015 [arXiv:1310.2248] [INSPIRE].

[4] A. Bartl, H. Eberl, K. Hidaka, T. Kon, W. Majerotto and Y. Yamada, Impact of squark pair decay modes on search for supersymmetric neutral Higgs bosons, Phys. Lett. B 389 (1996) 538 [hep-ph/9607388] [INSPIRE].

[5] M. Carena, S. Gori, N.R. Shah, C.E.M. Wagner and L.-T. Wang, Light Stops, Light Staus and the 125 GeV Higgs, JHEP 08 (2013) 087 [arXiv: 1303.4414] [INSPIRE].

[6] R.K. Barman, B. Bhattacherjee, A. Choudhury, D. Chowdhury, J. Lahiri and S. Ray, Current status of MSSM Higgs sector with LHC 13 TeV data, arXiv: 1608.02573 [INSPIRE].

[7] A. Djouadi, L. Maiani, A. Polosa, J. Quevillon and V. Riquer, Fully covering the MSSM Higgs sector at the LHC, JHEP 06 (2015) 168 [arXiv: 1502.05653] [INSPIRE].

[8] R.K. Barman, B. Bhattacherjee, A. Chakraborty and A. Choudhury, Study of MSSM heavy Higgs bosons decaying into charginos and neutralinos, Phys. Rev. D 94 (2016) 075013 [arXiv: 1607.00676] [INSPIRE]. 
[9] R.V. Harlander, S. Liebler and H. Mantler, SusHi: A program for the calculation of Higgs production in gluon fusion and bottom-quark annihilation in the Standard Model and the MSSM, Comput. Phys. Commun. 184 (2013) 1605 [arXiv: 1212.3249] [INSPIRE].

[10] R.V. Harlander, S. Liebler and H. Mantler, SusHi Bento: Beyond NNLO and the heavy-top limit, Comput. Phys. Commun. 212 (2017) 239 [arXiv:1605.03190] [INSPIRE].

[11] R.V. Harlander and W.B. Kilgore, Next-to-next-to-leading order Higgs production at hadron colliders, Phys. Rev. Lett. 88 (2002) 201801 [hep-ph/0201206] [INSPIRE].

[12] R.V. Harlander and W.B. Kilgore, Higgs boson production in bottom quark fusion at next-to-next-to leading order, Phys. Rev. D 68 (2003) 013001 [hep-ph/0304035] [INSPIRE].

[13] U. Aglietti, R. Bonciani, G. Degrassi and A. Vicini, Two loop light fermion contribution to Higgs production and decays, Phys. Lett. B 595 (2004) 432 [hep-ph/0404071] [INSPIRE].

[14] R. Bonciani, G. Degrassi and A. Vicini, On the Generalized Harmonic Polylogarithms of One Complex Variable, Comput. Phys. Commun. 182 (2011) 1253 [arXiv:1007.1891] [InSPIRE].

[15] G. Degrassi and P. Slavich, NLO QCD bottom corrections to Higgs boson production in the MSSM, JHEP 11 (2010) 044 [arXiv: 1007.3465] [INSPIRE].

[16] G. Degrassi, S. Di Vita and P. Slavich, NLO QCD corrections to pseudoscalar Higgs production in the MSSM, JHEP 08 (2011) 128 [arXiv:1107.0914] [INSPIRE].

[17] G. Degrassi, S. Di Vita and P. Slavich, On the NLO QCD Corrections to the Production of the Heaviest Neutral Higgs Scalar in the MSSM, Eur. Phys. J. C 72 (2012) 2032 [arXiv:1204.1016] [INSPIRE].

[18] R. Harlander and P. Kant, Higgs production and decay: Analytic results at next-to-leading order QCD, JHEP 12 (2005) 015 [hep-ph/0509189] [INSPIRE].

[19] K.G. Chetyrkin, J.H. Kuhn and M. Steinhauser, RunDec: A Mathematica package for running and decoupling of the strong coupling and quark masses, Comput. Phys. Commun. 133 (2000) 43 [hep-ph/0004189] [INSPIRE].

[20] A. Bartl, H. Eberl, K. Hidaka, T. Kon, W. Majerotto and Y. Yamada, QCD corrections to Higgs boson decays into squarks in the minimal supersymmetric standard model, Phys. Lett. B 402 (1997) 303 [hep-ph/9701398] [INSPIRE].

[21] H. Eberl, K. Hidaka, S. Kraml, W. Majerotto and Y. Yamada, Improved SUSY QCD corrections to Higgs boson decays into quarks and squarks, Phys. Rev. D 62 (2000) 055006 [hep-ph/9912463] [INSPIRE].

[22] F. Staub, SARAH, arXiv:0806.0538 [inSPIRE].

[23] F. Staub, SARAH 4: A tool for (not only SUSY) model builders, Comput. Phys. Commun. 185 (2014) 1773 [arXiv: 1309.7223] [inSPIRE].

[24] W. Porod, F. Staub and A. Vicente, A Flavor Kit for BSM models, Eur. Phys. J. C 74 (2014) 2992 [arXiv: 1405.1434] [INSPIRE].

[25] W. Porod, SPheno, a program for calculating supersymmetric spectra, SUSY particle decays and SUSY particle production at $e^{+} e^{-}$colliders, Comput. Phys. Commun. 153 (2003) 275 [hep-ph/0301101] [INSPIRE].

[26] W. Porod and F. Staub, SPheno 3.1: Extensions including flavour, CP-phases and models beyond the MSSM, Comput. Phys. Commun. 183 (2012) 2458 [arXiv:1104.1573] [INSPIRE].

[27] D. Straub et al., flav-io/flavio v0.21.2, May, (2017), https://doi.org/10.5281/zenodo.580247. 
[28] J.E. Camargo-Molina, B. O'Leary, W. Porod and F. Staub, Vevacious: A Tool For Finding The Global Minima Of One-Loop Effective Potentials With Many Scalars, Eur. Phys. J. C 73 (2013) 2588 [arXiv: 1307.1477] [inSPIRE].

[29] A. Djouadi, J. Kalinowski, P. Ohmann and P.M. Zerwas, Heavy SUSY Higgs bosons at $e^{+} e^{-}$ linear colliders, Z. Phys. C 74 (1997) 93 [hep-ph/9605339] [INSPIRE].

[30] M. Carena, S. Gori, I. Low, N.R. Shah and C.E.M. Wagner, Vacuum Stability and Higgs Diphoton Decays in the MSSM, JHEP 02 (2013) 114 [arXiv:1211.6136] [INSPIRE].

[31] W. Altmannshofer, M. Carena, N.R. Shah and F. Yu, Indirect Probes of the MSSM after the Higgs Discovery, JHEP 01 (2013) 160 [arXiv:1211.1976] [INSPIRE].

[32] L.A. Harland-Lang, A.D. Martin, P. Motylinski and R.S. Thorne, Parton distributions in the LHC era: MMHT 2014 PDFs, Eur. Phys. J. C 75 (2015) 204 [arXiv:1412.3989] [inSPIRE].

[33] H. Gomes, S. Gryb, T. Koslowski, F. Mercati and L. Smolin, A Shape Dynamical Approach to Holographic Renormalization, Eur. Phys. J. C 75 (2015) 3 [arXiv:1305.6315] [InSPIRE].

[34] C.L. Wainwright, CosmoTransitions: Computing Cosmological Phase Transition Temperatures and Bubble Profiles with Multiple Fields, Comput. Phys. Commun. 183 (2012) 2006 [arXiv: 1109.4189] [inSPIRE].

[35] T. Lee, T. Li and C. Tsai, Hom 4ps-2.0: A software package for solving polynomial systems by the polyhedral homotopy continuation method, Computing 83 (2008) 109.

[36] ATLAS and CMS collaborations, Measurements of the Higgs boson production and decay rates and constraints on its couplings from a combined ATLAS and CMS analysis of the LHC pp collision data at $\sqrt{s}=7$ and $8 \mathrm{TeV}$, JHEP 08 (2016) 045 [arXiv: 1606. 02266] [INSPIRE].

[37] ATLAS collaboration, ATLAS Run 1 searches for direct pair production of third-generation squarks at the Large Hadron Collider, Eur. Phys. J. C 75 (2015) 510 [arXiv:1506.08616] [INSPIRE].

[38] CMS collaboration, Search for direct production of bottom and top squark pairs in proton-proton collisions at $\sqrt{s}=13 \mathrm{TeV}$, CMS-PAS-SUS-16-032 [INSPIRE].

[39] CMS collaboration, Search for direct stop pair production in the dilepton final state at $\sqrt{s}=13 \mathrm{TeV}$, CMS-PAS-SUS-17-001 [INSPIRE].

[40] C. Borschensky et al., Squark and gluino production cross sections in pp collisions at $\sqrt{s}=13,14,33$ and $100 \mathrm{TeV}$, Eur. Phys. J. C 74 (2014) 3174 [arXiv:1407.5066] [InSPIRE].

[41] LHCb collaboration, Measurement of the $B_{s}^{0} \rightarrow \mu^{+} \mu^{-}$branching fraction and effective lifetime and search for $B^{0} \rightarrow \mu^{+} \mu^{-}$decays, Phys. Rev. Lett. 118 (2017) 191801 [arXiv: 1703.05747] [INSPIRE].

[42] Particle Data Group collaboration, C. Patrignani et al., Review of Particle Physics, Chin. Phys. C 40 (2016) 100001 [InSPIRE].

[43] W. Altmannshofer and D.M. Straub, New Physics in $B \rightarrow K^{*} \mu \mu$ ?, Eur. Phys. J. C 73 (2013) 2646 [arXiv: 1308.1501] [INSPIRE].

[44] M. Arana-Catania, The flavour of supersymmetry: Phenomenological implications of sfermion mixing, arXiv:1312.4888 [INSPIRE].

[45] M. Carena, J.R. Espinosa, M. Quirós and C.E.M. Wagner, Analytical expressions for radiatively corrected Higgs masses and couplings in the MSSM, Phys. Lett. B 355 (1995) 209 [hep-ph/9504316] [INSPIRE]. 
[46] J.D. Hunter, Matplotlib: A 2d graphics environment, Comput. Sci. Eng. 9 (2007) 90.

[47] M. Droettboom et al., matplotlib/matplotlib v2.0.2, May, (2017). https://doi.org/10.5281/zenodo.573577.

[48] J. Bezanson, S. Karpinski, V.B. Shah and A. Edelman, Julia: A Fast Dynamic Language for Technical Computing, arXiv:1209.5145.

[49] J. Bezanson, A. Edelman, S. Karpinski and V.B. Shah, Julia: A fresh approach to numerical computing, SIAM Review 59 (2017) 65 [arXiv:1411.1607]. 